\title{
Optimal Learning Behavior Prediction System Based on Cognitive Style Using Adaptive Optimization-Based Neural Network
}

\author{
Ghada Aldabbagh $\mathbb{D}^{1},{ }^{1}$ Daniyal M. Alghazzawi $\left(\mathbb{D},{ }^{1}\right.$ Syed Hamid Hasan, ${ }^{1}$ \\ Mohammed Alhaddad $\mathbb{D}^{1},{ }^{1}$ Areej Malibari $\mathbb{D}^{1},{ }^{1}$ and Li Cheng $\mathbb{D}^{2}$ \\ ${ }^{1}$ Faculty of Computing and Information Technology, King Abdulaziz University, P.O. Box. 80221, Jeddah-21589, Saudi Arabia \\ ${ }^{2}$ Xinjiang Technical Institute of Physics \& Chemistry Chinese Academy of Sciences, Ürümqi, China \\ Correspondence should be addressed to Daniyal M. Alghazzawi; dghazzawi@kau.edu.sa
}

Received 30 March 2020; Revised 4 September 2020; Accepted 30 September 2020; Published 5 November 2020

Academic Editor: Danilo Comminiello

Copyright (c) 2020 Ghada Aldabbagh et al. This is an open access article distributed under the Creative Commons Attribution License, which permits unrestricted use, distribution, and reproduction in any medium, provided the original work is properly cited.

\begin{abstract}
Widespread development of system software, the process of learning, and the excellence in profession of teaching are the formidable challenges faced by the learning behavior prediction system. The learning styles of teachers have different kinds of content designs to enhance their learning. In this learning environment, teachers can work together with the students, but the learning materials are designed by the teachers. The cognitive style deals with mental activities such as learning, remembering, thinking, and the usage of language. Therefore, being motivated by the problems mentioned above, this paper proposes the concept of adaptive optimization-based neural network (AONN). The learning behavior and browsing behavior features are extracted and incorporated into the input of artificial neural network (ANN). Hence, in this paper, the neural network weights are optimized with the use of grey wolf optimizer (GWO) algorithm. The output operation of e-learning with teaching equipment is chosen based on the cognitive style predicted by AONN. In experimental section, the measures of accuracy, sensitivity, specificity, time (sec), and memory (bytes) are carried out. Each of the measure is compared with the proposed AONN and existing fuzzy logic methodologies. Ultimately, the proposed AONN method produces higher accuracy, specificity, and sensitivity results. The results demonstrate that the algorithm proposed in this study can automatically learn network structures competitively, unlike those achieved for neural networks through standard approaches.
\end{abstract}

\section{Introduction}

With the progress and growth in Internet, there has been a rapid rise in the spread and dispensation of education [1]. The concept of e-learning does not mean only the provision of learning material to the potential learner on the web, but it also involves addressing the needs of instructors/teachers and learners/students who are seeking their own subjectrelated libraries. E-learning provides education to different learners at various levels irrespective of their learning needs, knowledge level, and preferences [2].

The learning process is fulfilled with the information of fresh interaction, self-regulation, and the individual knowledge of the students. The aim of the learning is to teach students until they gain complete access to the information they need. Important data are delivered by the enterprise resource planning (ERP) and learning management system (LMS) which aim to provide useful and wealthy data to the learners [3]. In the current age of globalization, appreciation of the quality of the education is the major process which is connected with the learning procedure. Eventually, schools require effective and better professionalism to improve student's knowledge via e-learning method. Effective use of concentration, sense organs, learning ways, and experiences by the students are the means of an educational society. The e-learning process is based on Internet which is the means of self-regulation. Behavior, motivation, and individual cognitive styles play significant role in the learning process. Exponential development of mobile system also provides e-learning service to the schools through their people, 
business, and employees [4]. Therefore, the learning object, model, content, and instruction are the core aspects of adaptive learning.

Colors, shapes, sizes, and surfaces of the objects appear different under different background and lighting conditions. It is difficult to distinguish the information related to the object while considering the image histogram that contains the information of the background and foreground [5]. The features of age estimation are extracted on the basis of the biometric inspired feature, anthropometric model, and the active appearance model [6]. It is a need of time to stimulate preliminary abstraction, recall procession, and local features to encode through encoding module. It is possible to minimize the sum of squared errors through inference outcome of the model and recommended outputs by multilayered perception and supervised learning rules [7].

A similar study conducted by Wiens [8] performed a predictive test through two-engine speed control. Further, it showed a lookup table based on the required engine speed and the current flow demand. There is a need to supply a specific electrical current to the coil, surrounded with magnetic field which is based on the desired setting pressure [9]. ANN is being widely accepted for different civil engineering realms, which plays an important role in structuring inner and outer information overflowing throughout the network [10]. There is a prerequisite of robust feature extraction and selection methods that is based on vision sensors for human interaction recognition (HIR).

The applications such as the security system, smart home system, surveillance system, and personal monitoring system are used for human activity recognition (HAR). The features of this system are useful in modeling, recognizing, and training daily activities. A new perspective of autonomous mobile robot path was presented by Jiang et al. [11] to propose a biased direction towards casual entropy maximization. The context information is unfolded and computational cost is reduced by dilated convolutions in convolutional neural denoising network (ECNDNet) [12].

Usually, classification and prediction methods are used to assess the academic performance in the research studies. The classification task is considered as a process to determine a model in which data were sorted into categories [13]. Neural networks are vital components of machine learning and are considered as one of the effective means to model classification issues that replicate neural activities of individuals. Neural networks are prepared with backpropagation learning algorithms, which are often slow and, therefore, require momentum and higher learning rates for accomplishing rapider convergence. These approaches work effectively only if the incremental preparation is needed; however, they are too modest to be practically integrated in real-life applications. Nevertheless, the Levenberg-Marquardt algorithm is still used for small- and medium-size networks [14]. The dearth of available information and memory impedes the use of faster and novel algorithms.

Linear and nonlinear issues are tackled through backpropagation which is a deterministic algorithm. However, backpropagation and its alternatives cannot provide comprehensive and vivid solutions. Another issue with backpropagation algorithms is the selection of adequate learning rate, which is a difficult concern. Unstable learning and excessively long training hours are the two major issues related to a linear network that are caused by too-fast learning rate and too-slow learning rate, respectively. The issue becomes further complicated for nonlinear multilayer networks as it is very complicated to find an easy approach to select a learning rate. For nonlinear networks, the error surface is also complicated as compared to linear networks [15]. However, several local minimum solutions in the error surface would be presented through neural networks with nonlinear transfer functions. Therefore, it is promising for a solution in a network to become stuck in a local condition. Additionally, the backpropagation learning algorithm does not produce perfect weight connections for the optimal solution. In this regard, the networks should be reinitialized concurrently to confirm that the effective solution has been obtained [16, 17].

Conversely, nature-based algorithms that are derived from animals' behavior are stochastic. Randomness is an important aspect of these algorithms. It indicates that initial randomized solutions are used in the algorithms that are then enhanced by using the sequence of iterations that prevent high local optima. Additionally, when it comes to deciding hidden neurons, a multilayer neural network is understated. There is a likelihood of the emergence of an underfitting issue when a minimal number of hidden neurons are utilized, and, similarly, overfitting may emerge when a large number of hidden neurons are utilized. Adaptive optimization-based neural network (AONN) is an alternative solution to a neural network prediction method. The human brain can be imitated by AONN for predicting student performance while considering the social and academic histories of students. This paper presents a combination of AONN and a modified grey wolf optimizer (GWO). The latter is utilized to optimize the former. Significantly, this method encourages the learning, education quantity, and the regulation of self-learning manner. There is also a need of providing theoretical analysis of supervised learning scenario in order to explain network architecture and parameters simultaneously. To the best of the researchers' knowledge, the current study is the first of its kind which focuses on generalization bounds for the problem of structural learning of neural networks to guide the design of a variety of different algorithms.

The primary aim of this paper is to design the effective e-learning process via adaptive optimization-based neural network (AONN) methodology. This paper aims to contribute to the extant literature in three ways. In the first place, the learning and browsing behavior of the learner is combined from these different cognitive styles and the recommended words are extracted effectively. In the second place, the neural network with training and testing phases is used to update the appropriate weights in the nodes using grey wolf optimizer (GWO). Finally, the proposed AONN method is implemented with the effectiveness of proposed 
methodology by using accuracy, specificity, sensitivity, time, and memory usage.

The rest of the paper is structured as follows. Section 2 discusses different e-learning styles related to previous works. Section 3 deliberates the learning and browsing behavior and proposes AONN methodology. Section 4 confers the experimental results based on AONN methodology and GWO algorithm. Finally, Section 5 presents the overall conclusions and recommendations for future studies.

\section{Related Work}

Learning styles can play an essential role in adaptive e-learning systems, which suggest preferred learning ways to students. The e-learning system can provide substantial advice and instructions for both teachers and students to optimize learning process. Furthermore, the e-learning system unlocks the platform for handling drawbacks of the conventional detection method that mainly uses questionnaire and statistical and computerized algorithms [18].

The lists of data are collected by using the learning tool of Felder and Soloman [19]. These tools are used to analyze and design various ways of learning and individual power and to figure out shortcomings in the learning manner. The process of organization, learning, and elaboration are the vital roles of cognitive concept. Trentin et al. [20] used networked collaborative learning (NCL) methods that are strongly implicated to the social collaborative learning and the activities of a network. However, the interrelation of teachers, learners, experts, and tutors is called a network.

Using a latent transition analysis of cognitive styles, Bouckenooghe et al. [21] identified several different cognitive style profiles including low-planning style profile, low creating style profile, a moderate cognitive style profile, and a dominant creative style profile. The study has also provided information of the trait-like character of these cognitive style profiles by explaining their temporal stability. In addition, significant differences emerge across profiles with respect to their relationship to distinct learning styles such as surface, strategic, and deep learning. According to Muhammad et al. [22], learning path refers to a systemic approach of learning objectives, which are designed to help students enhance their skill or knowledge in specific degree courses or subjects in online learning systems. Therefore, Muhammad et al. [22] reviewed the recent literature on learning path adaptation to fulfill two objectives: (1) to organize and examine the parameter of adaptation in learning path and (2) to discuss the issues encountered in adapting to learning path styles.

Further, considering their preferences, skills, and needs, Khamparia and Pandey [23] delivered a dynamic learning material to learners. Eight different learning attributes of student learning were classified by Khamparia and Pandey [23] on National Centre for Biotechnical Information (NCBI) e-learning database: student prior knowledge, personality, learning style, cognitive style, grades from previous semester, anxiety, motivation, and study level. A recent systematic review and meta-analysis on 129 articles have shown the effectiveness of adaptive and dynamic learning merged with different learning problems and styles [23]. The study has shown much more improvement in the performance and knowledge of learners in comparison with the conventional learning. Khamparia and Pandey [24] have also encouraged academicians, practitioners, and researchers to effectively adopt learning styles and strategies.

Mustafa and Gajawada [25] examined the self-assessment scores about the assumptions related to different brain dominance of learners with individual learning styles and differences. Blended learning is considered as influential approach of distance learning with respect to students' interaction, student/teacher interaction, and student's learning experience. Therefore, it has the potential to emerge as the predominant education model. Hasibuan et al. [26] carried out a knowledge generation process of research by using the latent semantic indexing method for identifying the personality traits that are possessed by each learner. Latent semantic indexing is a technique implemented to find meaning in a sentence via singular value decomposition. This system is used for generating the previous knowledge of each learner as a learner can forecast learning style while employing the artificial neural network method after collecting the previous knowledge.

Jalal and Mahmood [27] proposed a concept to embed cognitive procedures into information technologies for optimizing behaviors of students in virtual environments. The authors have identified student behaviors from the sequences of digital images using hybrid spatiotemporal features. Sensors and multimedia tools are used within e-learning methods to enhance resource sharing, interactivity, corporate trainings, and social networking. Xie et al. [28] examined various methods for developing learner profiles and determining the optimal method. The authors have collected feedback from ESL students in order to confirm the effectiveness of personalized work learning that was encouraged by the proposed model. The study has shown that the vocabulary acquisition can be better facilitated through the proposed learner profile model as compared to other benchmark methods.

Topuz et al. [29] collected the dataset of easy and hard learning manners of the learner's ability. However, the findings showed difference when they were compared with other learners. The continuous and constant manner predicts and stimulates the result of individual learning style. Mostly, the researchers focus on Myers-Briggs Type Indicator (MBTI) learning style to determine the cognitive style, individual learning, and the level of motivation. Huang et al. [24] introduced t-text book for the learners to improve their learning style and cognitive load. Most learners choose multimedia learning such as figures, charts, maps, pictures, and dynamic media with simulation.

Appel et al. [30] introduced the cognitive computing system by using the cognitive augmentation capacity to avoid the social issues. The data interfaces were represented as cognitive systems, and strength and weaknesses of each student were also verified. Zatarain et al. [31] suggested three 
different kinds of neural networks. In the presence of webbased environment, these approaches are used to detect the adaptive learning styles of the students. The most suitable learning styles are predicted with the help of fuzzy neural network and are classified by using genetic algorithm.

Moreover, the concept of extracting gait features of walking human from sequences of silhouette images was focused for recognition process [32]. One of the previous studies showed that spatial/temporal continuity, computed centroids of each activity, and constraints of human motion information are considered to be based on the chain coding mechanism and centroid point extraction [33]. Moreover, the augmented features are clustered and symbolized through self-organization maps to present each activity in a better way [34].

Huang et al. [35] proposed a robust adaptive sliding mode control scheme. It is under the simultaneous effect of parametric uncertainties and consistent external disturbance for altitude and attitude tracking of a quadrotor unnamed aerial vehicle (UAV) system. The underactuated dynamic model of the quadrotor UAV is initially developed through the Newton-Euler formalism. The controller is devised via sliding mode approach based on the nonlinear and strongly coupled attributes of the quadrotor. Moreover, the robustness of the proposed control scheme is enhanced through adaptive laws, alongside the parametric impairments of the system. It was confirmed that the control laws could minimize the attitude and altitude tracking blunders, even under a strong external disturbance.

The frequency control of microgrid becomes complicated because of stochastic power generations as well as dynamic uncertainties associated with the elevating renewable power generation. The energy storage system is often utilized in microgrid because it can offer flexible solutions for storing or releasing power energy. In this case, $\mathrm{Mu}$ et al. [36] proposed an intelligent control strategy on the basis of the adaptive dynamic programming for the frequency stability, which is devised for adjusting the power outputs of energy storage system and microturbine. In such situation, photovoltaic power generation is allied into the microgrid.

Zribi [37] proposed a novel adaptive tuning method of proportional-integral-derivative neural network controller for a nonlinear process. An improved gradient descent method has been utilized to adjust proportional-integralderivative neural network controller parameters in terms of external load disturbance and parameter alternatives. The effectiveness of the proposed algorithm has been confirmed through simulation findings with predefined learning methods. Hedjar [38] conducted simulations for showing the robustness of a neural inverse model. In addition, this adaptive neural inverse model controller is integrated into a temperature control system. Since improved tracking of performances had been acquired for different series point regulation, it was not affected by the large parameter variations and disturbances.

Notably, implementing neural networks that "learn to do implication" with rich model-building learning mechanisms can provide a promising way to explain human minds and understand the world. Therefore, the following section of this paper focuses on the behavioral structures of students' learning and browsing that are based on their cognitive styles.

\section{Material and Methods}

In this section, the behavioral structures of students' learning and browsing are identified and extracted on the basis of students' cognitive styles. A detailed explanation of the classification of these styles based on proposed AONN techniques is presented in the following sections.

3.1. Extraction of Learning Behavior. Various cognitive approaches were discussed based on the adaptive web interfaces. The web-based learning approach with cognitive style of the students and their paths has already been discussed by Silver et al. [37]. Based on the learning procedure, fifteen content and component interactions were selected by Silver et al. [37]. Northern Taiwan University with 176 sophomore students was chosen, and their behavior of browsing and cognitive manner relationships was examined.

Lo and Chan [38] examined the evaluation of cognitive style before the experiment based on questionnaires. During the process of web-based learning in a computer lab, the students were asked to browse for 30 minutes. Particularly, the learning data were not given in the classroom; otherwise, they never read the content before the trial. The test topics and the subtopics were stored online, and the user was asked to select and learn the topic for the test. Thereafter, the browsing performances of the students were noted in one system register file. From the register file, irregular data of 14 learners were ignored and the data of the remaining 162 contestants were examined. The various cognitive styles of the students such as mastery, thinking, understanding, feelings, interpersonal, intuition, and self-expression were categorized on the basis of their content selection process and component interactions. The web-based learning model of component interaction and the cognitive style relationships were identified through amassing interaction components such as content selection ratio and content staying time [37].

Ultimately, the adaptive interface of web-based approach with various students' cognitive styles of mastery, understanding, interpersonal, and self-expression were created on the basis of students' preference.

3.1.1. Mastery. This method focuses on the requirement of time by various students with similar materials and realized similar mastery levels. It contains teaching dissimilarity of the classic model and is mainly intended to check various abilities of the students.

3.1.2. Interpersonal. This style is entirely focused on the social situations and understanding ability of people. 
3.1.3. Understanding. The search process stimulates and improves the student's capacities to create usable proof, logics, and reasons. This process can be inspired with the remarkable development in the mysteries, evidences, troubles, and chances to debate and analyze.

3.1.4. Self-Expressive. In this process, the imagination and creation capacity of the students are highlighted. They introduce prototype, imagination, metaphor, direction of independency, and creativity.

3.2. Extraction of Browsing Behavior. The network adaptation is a fresh concept that enlarges the self-directed network concept and lets the stationary network move into dynamic environment of program through employing logic and intelligence. The experimentation accessibility needs the verification of adaptive e-learning and the collection of behavioral patterns, but the assembly of pattern behavior demands more efforts and time. Therefore, browsing the behavioral patterns helps in finding the students' learning model and pattern behavior. The browsing behavior of the test content was analyzed by using the followings steps.

3.2.1. Stop Word Removal. Generally, the search engine is programmed to delete the usual word of directory entries and recover the outcome of search query. Following four kinds of approaches were used for stop word removal:

3.2.2. Classic Approach. In this classic method, the precompiled register provides the stop words that are ignored.

3.2.3. Z-Approach. The Z-approach is also known as Zipf s law with the generation of three stop word methods that are stimulated to the additional list of classic stops. Therefore, highly frequent word ignorance is found only in one time such as the word of singleton. The low inverse text frequency ignorance is also considered in the Z-approach $[39,40]$.

3.2.4. Information of Mutual Approach. The shared information of the positive and negative classes of the document is computed while using shared informative approach. The proposal delivering number of information is informed regarding the known class. The proposal of low shared information with the delivering term contains less power discrimination which must be ignored [41, 42].

3.2.5. Term-Based Random Sampling (TBRS). Usually, the TBRS approach can identify the stop words from the web document. The division of larger piece of data is iterated and randomly chosen using TBRS. The Kullback-Leibler divergence is used to measure every larger piece of term position.

$$
\mathrm{d}_{y}(x)=P_{y}(x) \cdot \log _{2} \frac{p_{y}(x)}{P_{y}(x)} \mathrm{d}_{y}(x)=P_{y}(x) \cdot \log _{2} \frac{p_{y}(x)}{P_{y}(x)},
$$

where the term " $x$ " with normalized frequency is denoted as $P_{y}(x) P_{y}(x)$ in which the $y$ mass function can be utilized in the total collection of normalized frequency. Similarly, the entire duplications with all the least information such as larger space data were removed [37].

3.2.6. Stemming. The process of detecting the root or stem of the word is called as stemming. Every word such as attract, attracted, attracting, and attraction have been derived from the stem word "attract" [43]. The different suffixes and words are removed using stemming procedure which saves the space of memory and time. This paper used two key points for the implementation of stemming process. Firstly, the words were separated which never consisted of similar meaning. Secondly, the morphological representation of word was considered with similar fundamental meaning, and this can be mapped to the similar stem word.

3.2.7. Distinct Words. The distinct word contains same semantic understanding. The inflectional distinct words are connected with the syntactic changing of specified language such as gender, case, and plural. Where the word occurs, distinct words are connected with the sentence like part of speech [41]. Practically, the word "policy" contains different meanings, but the words "index" and "indices", which contain the same root, are never blended. The co-occurrences of different words are enhanced by using corpusbased stemming process.

3.2.8. Finding Meaning. Various sentences with similar word frequency and meanings are analyzed. It also identifies the meaning of the sentence and makes command in a single line [44]. Various locations with similar lexical method are connected to the dissimilar test. For example, lexically using schools: Ani sketches a school and Ani goes to school every day.

3.2.9. Singular Value Decomposition. The singular value decomposition method is the most popular factorization process. By the process of clustering, the node word weight and set of topic documents that deliver the words' frequency to the side of the theme are analyzed. Therefore, interlink between the words, tags, and documents are denoted the by graphs of double bipartite [45]. The singular value decomposition (SVD) method can destroy the correlation, cost of computational complexity, and noise. The dormant space can be received from the information using singular value decomposition. Therefore, the e-learning structure can be illustrated as shown in Figure 1. 


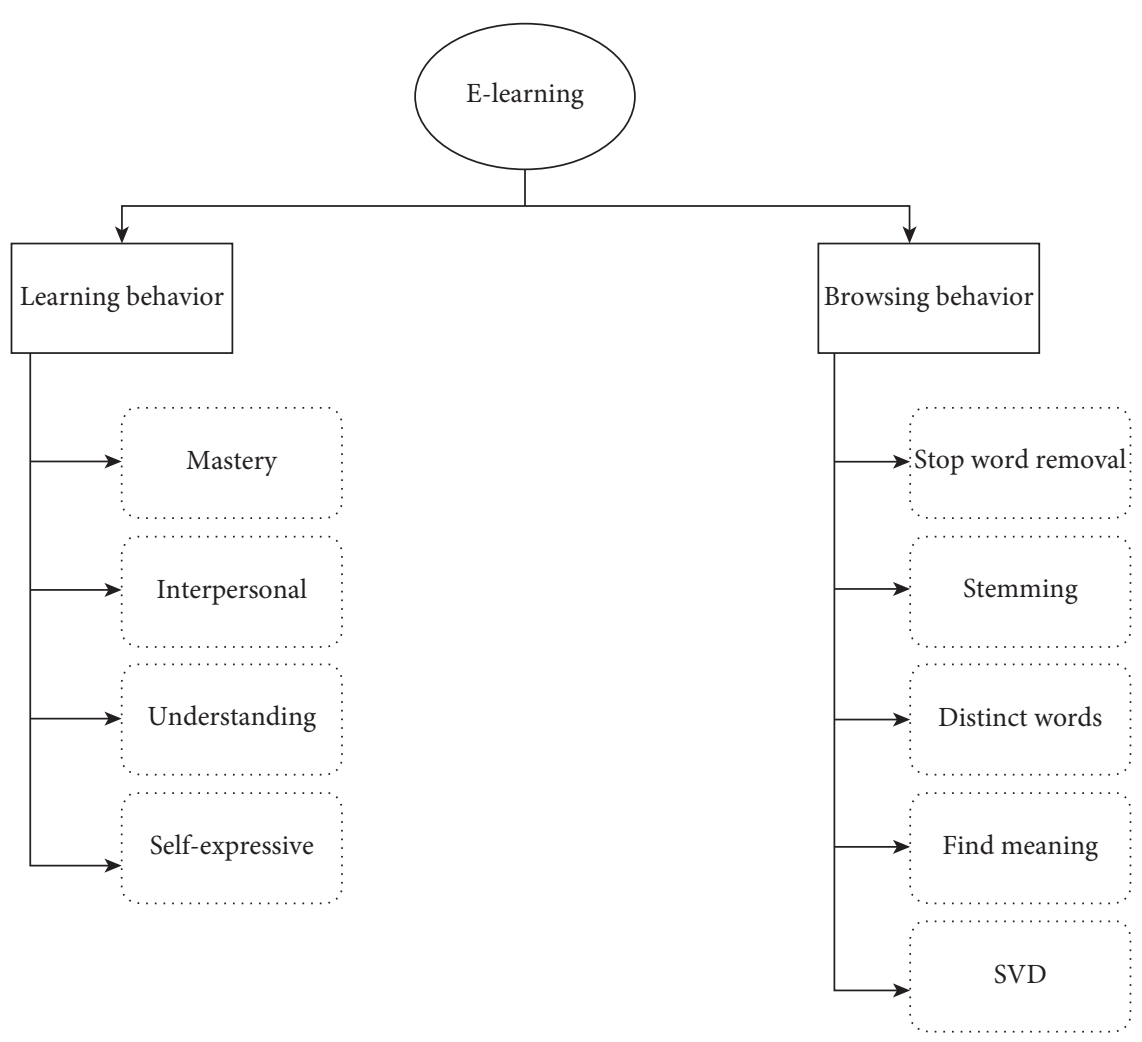

FIgURE 1: Structure of e-learning models.

3.3. Implementation of Proposed Adaptive OptimizationBased Neural Network (AONN). The computational architecture of artificial neural network (ANN) can be simulated by using biological nervous scheme. The neurons are simpler and mostly correlated with the ANN. Therefore, the neurons can be interrelated to next one through weighted associates to excessively pass the signals. The ANN procedures contain processing of data, analysis and collection, design structure of network, numeral hidden units and layers, initialization, network training, testing and simulation, and adjustment of weight. The procedure of data obtained from the dissimilar field using ANN is more helpful for fresh data prediction and detection. The computational architecture of ANN with unified processor is known as neurons that are interconnected by using weights. The computational information is fed into the learning and training processor. Significantly, the nonlinear interlink to the input and output is present with the usable data structures. Therefore, optimization, controlling of image, recognition of speech, estimation, and other host equipment are the application of ANNs. The real-world usage domains of ANN include business, mining, finance, and medical sectors [36]. Therefore, the ANN consists of training and testing phases (Figure 2).

3.3.1. Preprocessing. Consequent to collection, the dataset was normalized for processing and preparation through questionnaires. The data arrangement in a structure comprises five sets as cross-validation is used. Each set consists of an equal number of passing and failing students.

3.3.2. Feature Selection. This is an essential stage for classification. The most appropriate aspects were selected for classification. To be precise, the features that had no contribution to the classification output were removed. These features were evaluated through the correlation attribute in Weka between the features and the class. The dataset comprised 18 input characteristics and one output characteristic.

3.3.3. Classification. In the educational data mining field, several traditional classification algorithms are available. In this paper, AONN was used for the classification of browsing and learning behavior of learners after the completion of course. Details of the next stages are presented in the following sections.

3.3.4. Training Stage. The learning and browsing behavior of the learners was incorporated in the input for the neural network to train the network for the successful classification. Primarily, in a network, the weights are randomly allocated to every node. Hence, the output can be predefined in the training stage, but the outcome from the neural network can be used to estimate the original, and the weights are varied so it reduces the faults. This process is applicable for the prediction of larger number of learning styles, and it results in the system being stable, containing the weights as allocated in nodes. 


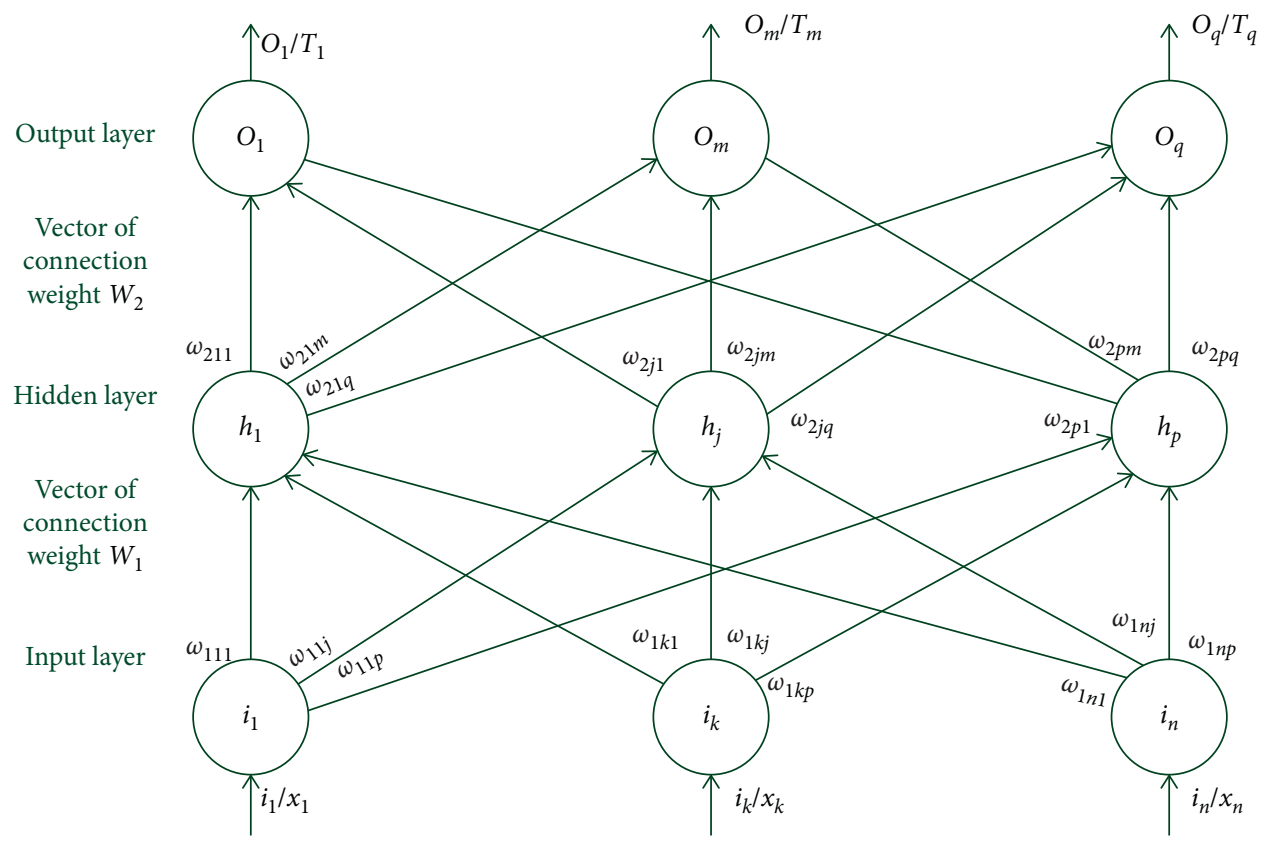

FIgURE 2: Computational architecture of ANN.

3.3.5. Testing Stage. After completing the training stage in a neural network, the next process is testing stage. Therefore, the input of learning and browsing behavior of the learners is given to the neural networks, which are updated using the suitable (respective) weights in the nodes. The output can be evaluated; however, the learning style classification depends on the trained dataset. The novelty of this method is shown from the optimized weights with the usage of grey wolf optimizer (GWO) algorithm [46].

3.3.6. Weight Optimization Using GWO Algorithm. A lot of grey wolves were sought and utilized for the prey idea in the GWO algorithm [47]. $\alpha, \beta$ and $\delta \alpha, \beta$ and $\delta$ assumed as the primary, secondary, and tertiary engineers of the wolf set. The outstanding wolves are noted with respect to $\omega \omega$ in order to use the procedure of GWO. The secondary and tertiary places of wolves are increasingly useful to the central wolf and delivered a few plans to prey chasing. The well-ordered procedure of GWO procedures are described in Algorithm 1. The preys that are surrounding, pursuing, and jumping are the most significant parts and tasks of the GWO algorithm.

The model structure is as follows: $18,10-10,10-10,1$ (18 neurons in the input layer, 10 neurons for the first context layer, 10 neurons in the first hidden layer, 10 neurons in the second hidden layer with 10 neurons for the second context layer, and 1 neuron in the output layer). Neurons from the previous time of the first and second hidden layers were imitated for the neurons of the first and second context layers, respectively. Following equations were proposed:

$$
C_{t}^{1}(t)=h_{j}^{1}(t-1)
$$

The $l$ th neuron in the first context layer at time $t$ is represented through $C_{t}^{1}(t)$. It is equal to $h_{j}^{1}(t-1)$, which portrays the $j$ th neuron in the first hidden layer at the previous time.

$$
C_{m}^{2}(t)=h_{g}^{2}(t-1)
$$

The $l$ th neuron in the second context layer at time $t$ is represented through $C_{m}^{2}(t)$. It is equal to $h_{g}^{2}(t-1)$, which portrays the $j$ th neuron in the second hidden layer at the previous time.

The following equation shows the feed-forward to the first hidden layer:

$$
\begin{aligned}
h_{j}^{1}(t) & =f\left(\sum_{i}^{I} V_{i j}^{1} x_{i}(t)\right)+f\left(\sum_{I}^{\text {Con }^{1}} U_{i j}^{1} C_{t}^{1}(t)\right), \\
f(\text { net }) & =\frac{1}{1+e^{- \text {net }}},
\end{aligned}
$$

where an activation function is represented through $f$ (net) in which both softmax and sigmoid are utilized for experimental objectives at the hidden layers in each hidden neuron and $V_{i j}^{1}$ and $U_{i j}^{1}$ represent weight connections related to the first hidden layer $h_{j}^{1}(t)$ and the input layer $x_{i}(t)$.

The following equation shows the feed-forward to the second hidden layer:

$$
h_{g}^{2}(t)=f\left(\sum_{j}^{H 1} V_{j g}^{2} h_{j}^{1}(t)\right)+f\left(\sum_{m}^{\mathrm{Con}^{2}} U_{m g}^{2} C_{m}^{2}(t)\right),
$$


where connections between the first layer $h_{j}^{1}(t)$ and the second hidden layer $h_{g}^{2}(t)$ and between the second context layer $C_{m}^{2}(t)$ and the second hidden layer $h_{g}^{2}(t)$ are represented through $V_{j g}^{2}$ and $U_{m g}^{2}$.

3.3.7. Encircling of Prey. The encompassed idea is grasped for pursuing the cycle-based scientific activities on the specific conditions. Hence, the vector coefficients $\bar{D}$ and $\overline{E D}$ and $\bar{E}$ are inferred by $\bar{D}=2 \bar{d} \cdot r_{1}-\bar{d} \bar{D}=2 \bar{d} \cdot \overline{r_{1}}-\bar{d}$ and $\bar{E}=2 \cdot \overline{r_{2}} \bar{E}=2 \cdot \bar{r}_{2}$ where $\vec{r}_{1}$ and $\vec{r}_{2}$ vectors have a common place with the interim $[0,1]$. The vector estimation of $\bar{d}=d_{1}\left(1-\left(i / i_{\max }\right)\right) \bar{d}=d_{1}\left(1-\left(i / i_{\max }\right)\right)$ is reduced from 0 to greatest number of $i_{\max } i_{\max }$ iterations.

$$
\begin{aligned}
\bar{C} & =\left|\bar{E}_{Y_{r}}(i)-\bar{Y}_{(i)}\right| \bar{Y}(i+1) \\
& =\bar{Y}_{r}(i)-\bar{D} \cdot \overline{C C} \\
& =\left|\bar{E} \cdot \bar{Y}_{r}(i)-\bar{Y}_{(i)}\right|, \\
\bar{Y}(i+1) & =\bar{Y}_{r}(i)-\bar{D} \cdot \bar{C} .
\end{aligned}
$$

3.3.8. Hunting. The chief $\alpha, \beta$ and $\delta \alpha, \beta$ and $\delta$ solutions are required for the $\beta$ and $\delta \beta$ and $\delta$ GWO. The top most arrangement positions to be put away in the bundle and the other estimation of wolves $\omega \omega$ can be updated on the matched location. The mathematical clarification of the updated positions is presented in the accompanying conditions.

$$
\begin{aligned}
\bar{C}_{\alpha} & =\left|\bar{E}_{1} \cdot \bar{Y}_{\alpha}(i)-\bar{Y}\right| \\
\bar{C}_{\beta} & =\left|\bar{E}_{2} \cdot \bar{Y}_{\beta}(i)-\bar{Y}\right| \\
\bar{C}_{\delta} & =\left|\bar{E}_{3} \cdot \bar{Y}_{\delta}(i)-\bar{Y}\right| \bar{Y}_{I}=\bar{Y}_{\alpha}(i)-\bar{D}_{I} \cdot\left(\bar{C}_{\alpha}\right), \\
\bar{Y}_{2} & =\bar{Y}_{\beta}(i)-\bar{D}_{I} \cdot\left(\bar{C}_{\beta}\right) \bar{Y}(i+1) \\
& =\frac{\bar{Y}_{I}+\bar{Y}_{2}+\bar{Y}_{3}}{3} \bar{C}_{\alpha}\left|\bar{E}_{1} \cdot \bar{Y}_{\alpha}(i)-\bar{Y}\right| \\
\bar{C}_{\beta}= & \left|\bar{E}_{2} \cdot \bar{Y}_{\beta}(i)-\bar{Y}\right| \\
\bar{C}_{\delta}= & \left|\bar{E}_{3} \cdot \bar{Y}_{\delta}(i)-\bar{Y}\right| \\
\bar{Y}_{I}= & \bar{Y}_{\alpha}(i)-\bar{D}_{I} \cdot\left(\bar{C}_{\alpha}\right) \\
\bar{Y}_{2}= & \bar{Y}_{\beta}(i)-\bar{D}_{I} \cdot\left(\bar{C}_{\beta}\right) \\
\bar{Y}(i+1)= & \frac{\bar{Y}_{I}+\bar{Y}_{2}+\bar{Y}_{3}}{3} .
\end{aligned}
$$

3.3.9. Pouncing. The changeable value $\mathrm{d}$ commands with the stage of GWO and the rate winds up distorted by its iterative location. The prey chasing is constrained by the vectors, $\vec{D}$, $\vec{E}$ and the $\vec{D}$ vector esteem distorted from - 2 d to $2 \mathrm{~d}$ with $|\vec{D}|<1$ for the pouncing of prey (exploitation) and the prey looking (exploration) can further devise methods income $|\vec{D}|>1$.

The major problem is the metaheuristic in the training stage of ANN, and it is overcome by using metaheuristic algorithm. Therefore, a GWO algorithm is introduced for updating weights of ANN. The single hidden layer of MLP network with most popular variable weights and biases is optimized using GWO. The signal link between two neurons is calculated by using this method. The trainer must identify the weights and biases of set values. The classification accuracy of each grey wolf updates can set the fitness values based on a random interval $[0,1]$. It is updated when the fitness function meets the suitable accuracy of classification, and the vector positions are stored and provide better ANN learning outcome. The AONN delivers the result of better suitable accuracy prediction and classification, which collects the student model and the adaption model. The AONN can train automatically and dynamically to identify the student learning styles by considering the customized learning materials to the learners. The proposed AONN methodology can design adaptive web interfaces of students' cognitive style and can also examine the dealings between the students' cognitive style and content browsing pattern as well as the component interaction (Figure 3).

\section{Results and Discussion}

The data regarding learning and browsing behavior of students with cognitive style were collected and trained using AONN. This paper has analyzed the proposed AONN method with other existing concepts for the purpose of obtaining better results. Result data of five different tests were used for the experimental analysis. Therefore, the performance of the proposed AONN of the e-learning method has been compared with existing fuzzy logic The proposed AONN is evaluated on a desktop computer with Intel ${ }^{\circledR}$ Core $3.91 \mathrm{GHz}$ along with $8 \mathrm{~GB}$ memory with the codes running in JAVA software.

The samples were initially fed into the AONN, which is followed by a reflection of the output from the first hidden layer at time $t$ being held in the first context layer. Afterwards, the net is fed back to the first hidden layer at time $t+1$. Correspondingly, a reflection of the output was held back in the second context layer from the second hidden layer at time $t$. Next, the net was fed back to the second hidden layer at time $t+1$. A set of state summarizing previous inputs was preserved and learned through the AONN model. This procedure was repeated iteratively to feed all the other training samples into the AONN using the same initialized biases and weights. The vector of biases and weights was adjusted iteratively on the basis of the number of search agents in terms of alpha, beta, and gamma after the fitness and the position of each of the best modernized wolves were analyzed. The weights and biases are passed to the AONN after they are updated in the AONN.

4.1. Evaluation Metrics. This paper has collected data from the students of Northern Taiwan University. The e-learning model of the proposed AONN performance has been 


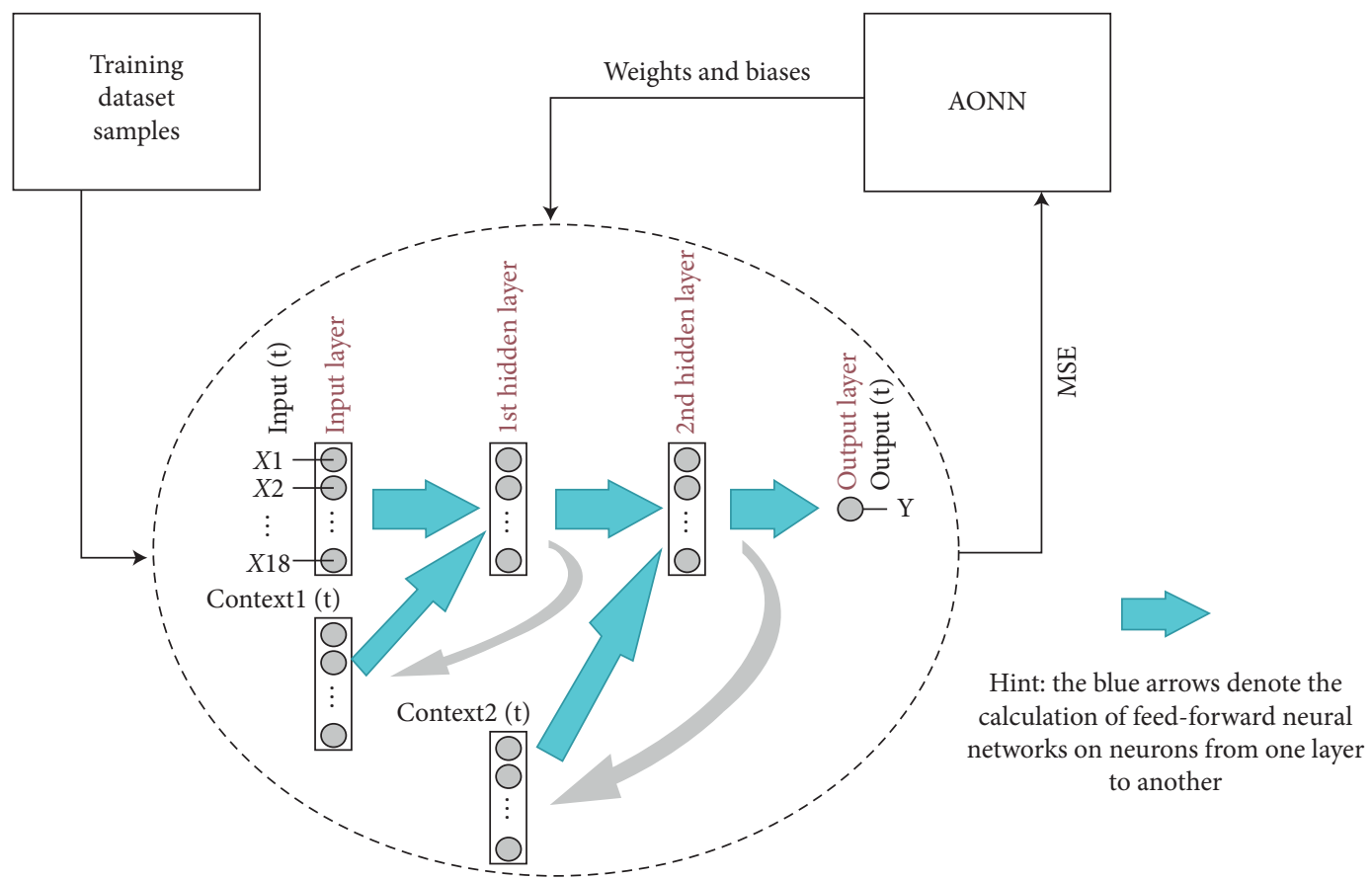

FIgURE 3: GWO algorithm output.

analyzed with various tests. Initially, five-test result data were compared with the proposed AONN and fuzzy method. Afterwards, the performance evaluation of various measures with excess confidential occurrence was performed. The test data with various factors such as accuracy, sensitivity, specificity, memory usage, and time are discussed in the following section. The positive true (PT), negative true $(\mathrm{NT})$, positive false (FP), and negative false (NP) are the performance criteria for the classification of test results.

The students' learning style with five training test data was taken for the analysis of accuracy performance. The accuracy test delivered the correct prediction results. Therefore, the test data were compared with measure of accuracy to give better accuracy result in learning in comparison with that of the fuzzy logic. Hence, the accuracy performance was evaluated using equation (10). Ultimately, Figure 4 shows the enhanced accuracy result of the proposed AONN in each test when compared with existing fuzzy logic.

$$
A=\frac{\mathrm{PT}+\mathrm{NT}}{(\mathrm{PT}+\mathrm{NT}+\mathrm{PF}+\mathrm{NF})} A=\frac{\mathrm{PT}+\mathrm{NT}}{(\mathrm{PT}+\mathrm{NT}+\mathrm{PF}+\mathrm{NF})}
$$

Usually, the sensitivity test is carried out for the performance evaluation of learners' learning style. The true positive cases are classified using equation (11). The comparison results of proposed AONN with existing fuzzy in each test can yield the suitable higher sensitivity results. Compared to existing results, the proposed AONN contained more sensitivity ideas to observe the subject, which is shown in Figure 5.

$$
S_{\mathrm{nt}}=\frac{\mathrm{PT}}{(\mathrm{PT}+\mathrm{NF})} S_{\mathrm{nt}}=\frac{\mathrm{PT}}{(\mathrm{PT}+\mathrm{NF})} .
$$

The students' learning style with five training test data was chosen for the analysis of specificity performance. The specificity is the process to identify the negative individuals. The accuracy test delivered the correct prediction results. Hence, the test data were compared with the measure of specificity, which provided excellent outcomes in learning when compared to the fuzzy logic. Ultimately, Figure 6 delineated the specificity result of proposed AONN in each test, which is compared with existing fuzzy logic. Hence, the performance of specificity was evaluated using the following equation:

$$
S_{\mathrm{pcf}}=\frac{\mathrm{TN}}{(\mathrm{TN}+\mathrm{PF})} S_{\mathrm{pcf}}=\frac{\mathrm{TN}}{(\mathrm{TN}+\mathrm{PF})} .
$$

The use and learning behavioral model of the proposed AONN will drastically reduce the time required for learning the subject; on the other hand, the followers of existing fuzzy method take more time to learn the subject. The less time requirement of the proposed AONN is shown in Figure 7. The learners using the proposed AONN learned the topics quickly or within few seconds in every test.

The memory usage is the major part of learning process. The user of the learning model of the proposed AONN will use minimum memory to learn the topics; the followers of the existing fuzzy method will use more memory to learn the subject. The less memory space usage of proposed AONN is shown in Figure 8. The learners using the proposed AONN learned the topic quickly or within few seconds in every test. 


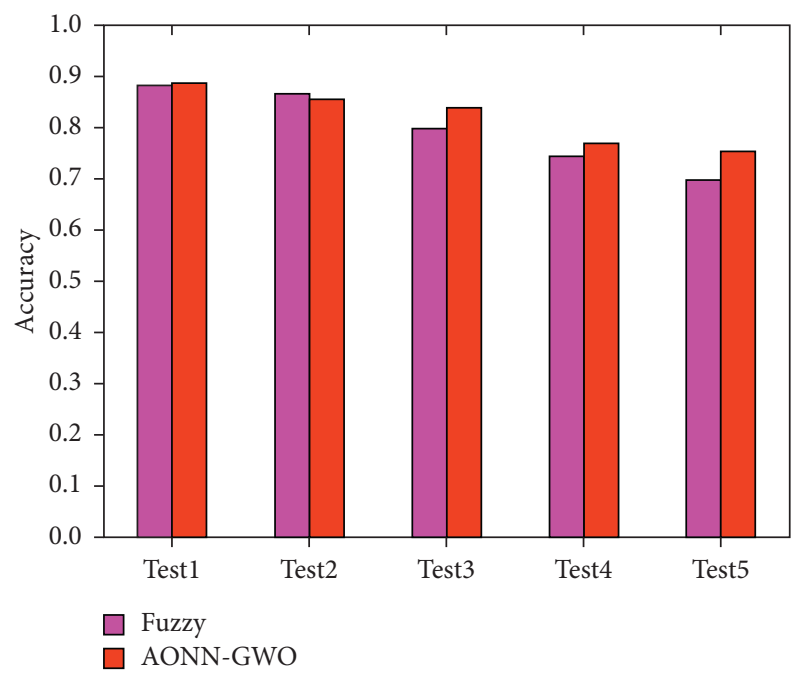

Figure 4: Comparative analysis of accuracy result.

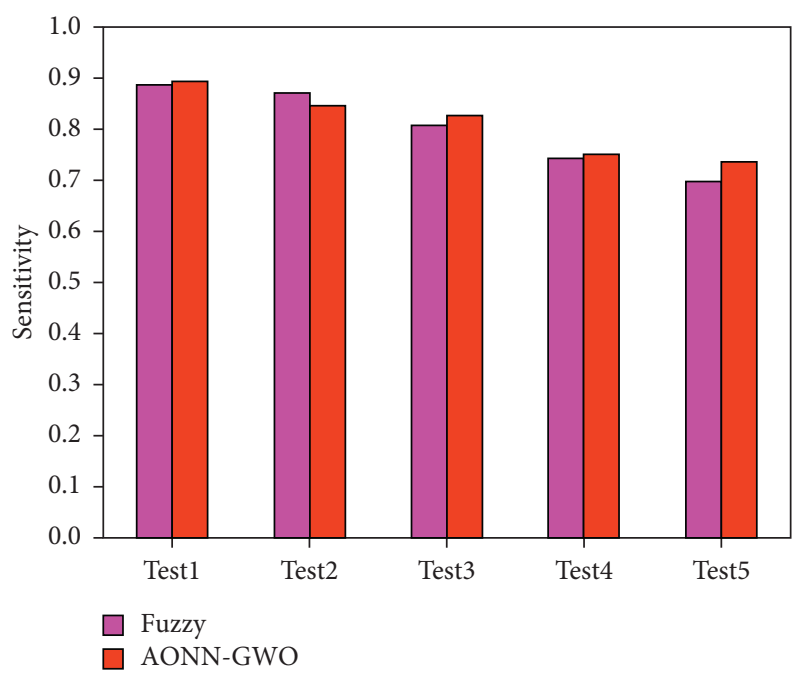

FIgURE 5: Comparative analysis of sensitivity result.

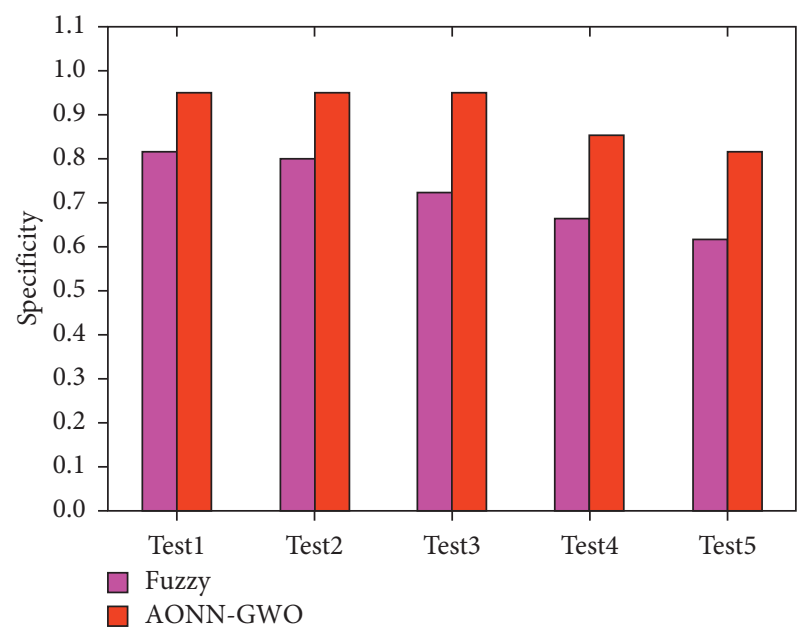

FIgUre 6: Comparative analysis of specificity result.

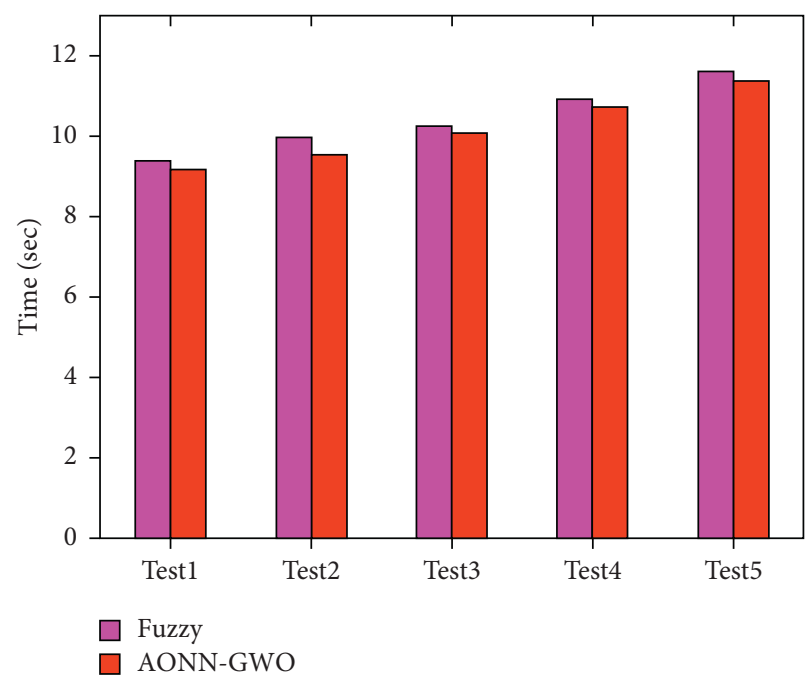

Figure 7: Comparative analysis of time result.

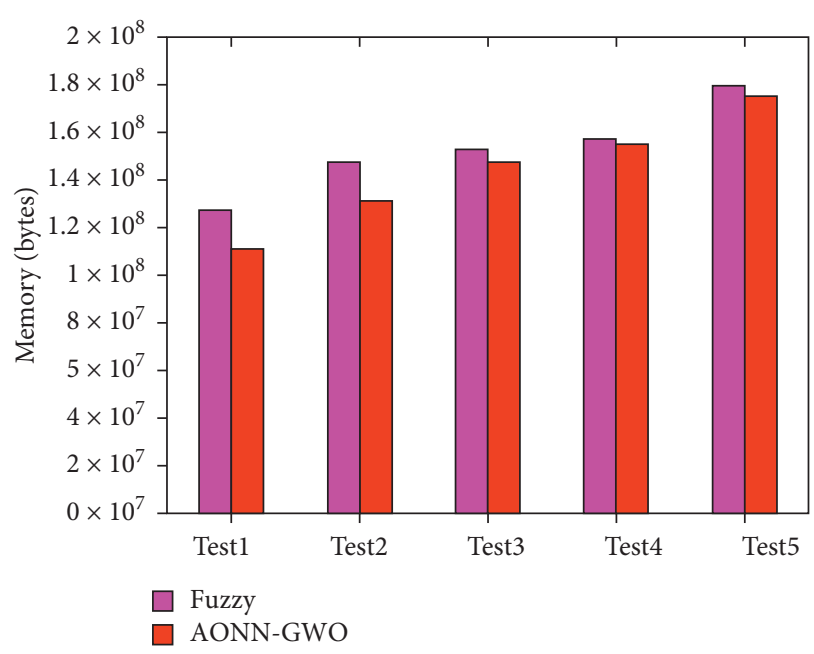

FIGURE 8: Comparative analysis of time result. 
Input: search population variable $S_{m}$, solution size $a$, the solution from upper to lower limit

$\left[V a_{1}, \ldots, V a_{n}, N a_{1}, \ldots, N a_{n}\right]$, maximum iteration

Output: the topmost pounce factor $\vec{Y}_{\alpha}$

(1) Initialize the population of grey wolf solution $\vec{Y}_{p}=\left(y_{1}, y_{2}, \ldots, y_{n}\right)$ where $p \in\left[S_{m}\right]$ and $y_{q} \in\left[V a_{q}, N a_{q}\right] \forall \forall_{q} \in[a]$

(2) Initialize $d, \vec{D}, \vec{E}$, and $i=1$

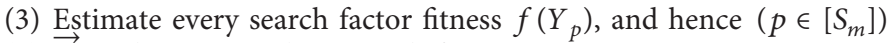

(4) $\vec{Y}_{\alpha}=$ the primary best search factor

(5) $\vec{Y}_{\beta}^{\alpha}=$ the secondary best search factor

(6) $\vec{Y}_{\delta}=$ the tertiary best search factor

(7) while ( $i<$ maximum iteration) do

(8) for every search factor do

(9) Upgrade the current search factor location using equation (9)

(10) end for

(11) Upgrade $d, \vec{D}$, and $\vec{E}$

(12) Estimate every search factor fitness

(13) Upgrade $\vec{Y}_{\alpha}, \vec{Y}_{\beta}$, and $\vec{Y}_{\delta}$

(14) $i=i+1$

(15) end while

(16) return $\vec{Y}_{\alpha}$

Algorithm 1: Grey wolf optimizer algorithm.

TABLE 1: Overall performance result of AONN.

\begin{tabular}{lccccc}
\hline $\begin{array}{l}\text { Test/ } \\
\text { measures }\end{array}$ & Accuracy & Sensitivity & Specificity & $\begin{array}{c}\text { Time } \\
(\mathrm{sec})\end{array}$ & Memory \\
\hline Test-1 & 0.888 & 0.8936 & 0.9545 & 9.142 & 111183944 \\
Test-2 & 0.854166 & 0.8444 & 0.95 & 9.567 & 131062556 \\
Test-3 & 0.8367 & 0.82608 & 0.95 & 10.12 & 146654264 \\
Test-4 & 0.7647 & 0.75 & 0.85714 & 10.658 & 154896522 \\
Test-5 & 0.75 & 0.73469 & 0.81818 & 11.343 & 175605560 \\
\hline
\end{tabular}

TABLE 2: Comparison of the proposed system with conventional systems with respect to accuracy.

\begin{tabular}{lc}
\hline Systems & Accuracy (\%) \\
\hline AONN & 85.9 \\
MVDR & 71.23 \\
Human activity recognition rate & 80.05 \\
Hidden Markov model & 63.7 \\
Depth silhouette-based ridge data [5] & 81.17 \\
Ambient home smart system & 85.17 \\
\hline
\end{tabular}

4.2. Overall Performance of Proposed AONN in E-Learning. Finally, the students of Northern Taiwan University with each test data went through various tests such as accuracy, specificity, sensitivity, time (sec), and memory usage. In all cases, the measures were compared with proposed AONN and existing fuzzy logic. The proposed AONN delivered the result of higher accuracy, specificity, and sensitivity in the entire test, and the capacity of time and memory usage was low when compared to the existing method. Therefore, the proposed AONN delivered suitable results in terms of accuracy, specificity, sensitivity, time, and memory. The entire performance of proposed AONN is presented in Table 1.
4.3. Accuracy-Based Comparison of Proposed System with Conventional Systems. The proposed system (AONN) based on GWO algorithm delivered superior accuracy rate (85.9\%) for learning and browsing behavior. Their proposed system showed $71.23 \%$ accuracy of speech recognition. They recommended that the proposed system must be implemented in e-healthcare systems. These systems aim to monitor elderly individuals as an indoor environment system meant to identify the activities of multiple users. Also, these systems are used as surveillance systems to observe pedestrian traffic areas. Likewise, Jalal et al. [5] showed $81.17 \%$ accuracy for recognizing body part that was posed by undertaking depth silhouette-based ridge data (Table 2).

\section{Conclusion}

The efficient learning and browsing behavior features were extracted and classified using ANN with GWO for weight updates. Thereafter, the process of training and testing automatically identified the students' cognitive styles by using proposed AONN. The customized learning materials were received with the help of AONN. The adaptive web interfaces of AONN identified the relationship between students' cognitive styles and browsing patterns of interactive components and content. The AONN methodology has been implemented by using JAVA software. Experimentally, the proposed AONN and existing fuzzy models were compared in terms of different measures such as accuracy, specificity, sensitivity, time (sec), and memory (bytes). Nonetheless, the proposed AONN provided the result showing higher ranges of accuracy, specificity, and sensitivity and lower ranges of time and memory usage in comparison with the existing method. Therefore, the proposed AONN methodology was proved to be more effective in terms of e-learning. 


\section{Data Availability}

The data used to support the findings of the study are available from the corresponding author upon request.

\section{Conflicts of Interest}

The authors declare that they have no conflicts of interest.

\section{Acknowledgments}

The authors are thankful to all the associated personnel, who contributed to this study by any means. This project was funded by the National Plan for Science, Technology and Innovation (MAARIFAH), King Abdulaziz City for Science and Technology, the Kingdom of Saudi Arabia, award number 12-INF2259-03. The authors also acknowledge with thanks the Science and Technology Unit, King Abdulaziz University, for technical support.

\section{References}

[1] K. Chrysafiadi and M. Virvou, "Evaluating the integration of fuzzy logic into the student model of a web-based learning environment," Expert Systems with Applications, vol. 39, no. 18, pp. 13127-13134, 2012.

[2] A. Kumar, N. Singh, and N. Jyothi-Ahuja, "Learning styles based adaptive intelligent tutoring systems: document analysis of articles published between 2001 and 2016 and 2017," International Journal of Cognitive Research in Science, Engineering and Education, vol. 5, no. 2, pp. 83-97, 2017.

[3] P. Berking and S. Gallagher, Choosing a Learning Management System, pp. 1-136, Advanced Distributed Learning (ADL) Co-Laboratories, Alexandria, VA, USA, 2016.

[4] A. Y.-L. Chong, "Understanding mobile commerce continuance intentions: an empirical analysis of Chinese consumers," Journal of Computer Information Systems, vol. 53, no. 4, pp. 22-30, 2013.

[5] S. Susan, P. Agrawal, M. Mittal, and S. Bansal, "New shape descriptor in the context of edge continuity," CAAI Transactions on Intelligence Technology, vol. 4, no. 2, pp. 101-109, 2019.

[6] Y. Tingting, W. Junqian, W. Lintai, and X. Yong, "Three-stage network for age estimation," CAAI Transactions on Intelligence Technology, vol. 4, no. 2, pp. 122-126, 2019.

[7] C. Zhu and D. Miao, "Influence of kernel clustering on an RBFN," CAAI Transactions on Intelligence Technology, vol. 4, no. 4, pp. 255-260, 2019.

[8] T. Wiens, "Engine speed reduction for hydraulic machinery using predictive algorithms," International Journal of Hydromechatronics, vol. 2, no. 1, p. 16, 2019.

[9] S. Osterland and J. Weber, "Analytical analysis of single-stage pressure relief valves," International Journal of Hydromechatronics, vol. 2, no. 1, p. 32, 2019.

[10] M. Shokri and K. Tavakoli, "A review on the artificial neural network approach to analysis and prediction of seismic damage in infrastructure," International Journal of Hydromechatronics, vol. 2, no. 4, p. 178, 2019.

[11] R. Jiang, H. Zhou, H. Wang, and S. S. Ge, "Maximum entropy searching," CAAI Transactions on Intelligence Technology, vol. 4, no. 1, pp. 1-8, 2019.
[12] C. Tian, Y. Xu, L. Fei, J. Wang, J. Wen, and N. Luo, "Enhanced CNN for image denoising," CAAI Transactions on Intelligence Technology, vol. 4, no. 1, pp. 17-23, 2019.

[13] J. Han, J. Pei, and M. Kamber, Data Mining: Concepts and Techniques, Elsevier, Amsterdam, Netherlands, 2011.

[14] T. A. Rashid, D. K. Abbas, and Y. K. Turel, "A multi hidden recurrent neural network with a modified grey wolf optimizer," PLoS One, vol. 14, no. 3, Article ID e0213237, 2019.

[15] M. T. Hagan, H. B. Demuth, and M. H. Beale, Neural Network Design, Pws Publishing, Boston, MA, USA, 1996.

[16] M. F. Sikder, M. J. Uddin, and S. Halder, "Predicting students yearly performance using neural network: a case study of BSMRSTU," in Proceedings of the 2016 5th International Conference On Informatics, Electronics And Vision (ICIEV), Dhaka, Bangladesh, May 2016.

[17] B. Kumar and S. Pal, "Mining educational data to analyze students performance," International Journal of Advanced Computer Science And Applications, vol. 2, no. 6, 2011.

[18] H. M. Truong, "Integrating learning styles and adaptive e-learning system: current developments, problems and opportunities," Computers in Human Behavior, vol. 55, pp. 1185-1193, 2016.

[19] R. M. Felder and B. A. Soloman, Learning Styles and Strategies, Richmond Publishing, London, UK, 2000.

[20] G. Trentin, Networked Collaborative Learning: Social Interaction and Active Learning, Elsevier, Amsterdam, Netherlands, 2010.

[21] D. Bouckenooghe, E. Cools, D. De Clercq, K. Vanderheyden, and T. Fatima, "Exploring the impact of cognitive style profiles on different learning approaches: empirical evidence for adopting a person-centered perspective," Learning and Individual Differences, vol. 51, pp. 299-306, 2016.

[22] A. Muhammad, Q. Zhou, G. Beydoun, D. Xu, and J. Shen, "Learning path adaptation in online learning systems," in Proceedings of the 2016 IEEE 20th International Conference On Computer Supported Cooperative Work In Design (CSCWD), Wellington, New Zealand, April 2016.

[23] A. Khamparia and B. Pandey, "Association of learning styles with different e-learning problems: a systematic review and classification," Education and Information Technologies, vol. 25, no. 2, pp. 1303-1331, 2019.

[24] K.-L. Huang, K.-H. Chen, and C.-H. Ho, "Enhancing learning outcomes through new e-textbooks: a desirable combination of presentation methods and concept maps," Australasian Journal of Educational Technology, vol. 30, no. 5, 2014.

[25] H. M. Mustafa and S. Gajawada, "Application of artificial neural networks modeling for self-assessment performance evaluation regarding blended learning paradigm (brain based approach)," American Journal Of Engineering Research (AJER) E-ISSN, vol. 2, no. 2, pp. 2320-0847, 2017.

[26] M. S. Hasibuan, L. E. Nugroho, and P. I. Santosa, "Model detecting learning styles with artificial neural network," Journal of Technology and Science Education, vol. 9, no. 1, p. 85, 2019.

[27] A. Jalal and M. Mahmood, "Students' behavior mining in e-learning environment using cognitive processes with information technologies," Education and Information Technologies, vol. 24, no. 5, pp. 2797-2821, 2019.

[28] H. Xie, D. Zou, R. Zhang, M. Wang, and R. Kwan, "Personalized word learning for university students: a profilebased method for e-learning systems," Journal of Computing in Higher Education, vol. 31, no. 2, pp. 273-289, 2019.

[29] C. Topuz and Z. Arasan, "Kaizen-educational: an awarenessraising and motivational-enhancement group counseling 
model," Procedia - Social and Behavioral Sciences, vol. 84, pp. 1356-1360, 2013.

[30] A. P. Appel, H. Candello, and F. L. Gandour, "Cognitive computing: where big data is driving us," Handbook of Big Data Technologies, Springer, Berlin, Germany, pp. 807-850, 2017.

[31] R. Zatarain-Cabada, M. L. Barrón-Estrada, L. ZepedaSánchez, G. Sandoval, J. M. Osorio-Velazquez, and J. E. UriasBarrientos, "A kohonen network for modeling students' learning styles in web 2.0 collaborative learning systems," Lecture Notes in Computer Science, Springer, Berlin, Germany, pp. 512-520, 2009.

[32] N. I. Yaacob and N. M. Tahir, "Feature selection for gait recognition," in Proceedings of the 2012 IEEE Symposium On Humanities, Science and Engineering Research, Kuala Lumpur, Malaysia, June 2012.

[33] T. Huang, D. Huang, Z. Wang, and A. Shah, "Robust tracking control of a quadrotor UAV based on adaptive sliding mode controller," Complexity, vol. 2019, Article ID 7931632, 15 pages, 2019.

[34] C. Mu, Y. Zhang, H. Jia, and H. He, "Energy-storage-based intelligent frequency control of microgrid with stochastic model uncertainties," IEEE Transactions on Smart Grid, vol. 11, no. 2, pp. 1748-1758, 2020.

[35] A. Zribi, M. Chtourou, and M. Djemel, "A new PID neural network controller design for nonlinear processes," Journal of Circuits, Systems and Computers, vol. 27, no. 4, Article ID 1850065, 2017.

[36] R. Hedjar, "Online adaptive control of non-linear plants using neural networks with application to temperature control system," Journal of King Saud University - Computer and Information Sciences, vol. 19, pp. 75-94, 2007.

[37] H. F. Silver, R. W. Strong, and M. J. Perini, So, Each May Learn: Integrating Learning Styles and Multiple Intelligences, pp. 22311-31714, Association for Supervision and Curriculum Development, Alexandria, VA, USA, 2000.

[38] J.-J. Lo and Y.-C. Chan, "Relationships between user cognitive styles and browsing behaviors of an online learning web site," in Proceedings of the 2008 International Conference on Cyberworlds, Hangzhou, China, September 2008.

[39] A. G. Jivani, "A comparative study of stemming algorithms," International Journal of Computer Applications in Technology, vol. 2, no. 6, pp. 1930-1938, 2011.

[40] D. Sharma, "Stemming algorithms: a comparative study and their analysis," International Journal of Applied Information Systems, vol. 4, no. 3, pp. 7-12, 2012.

[41] S. Vijayarani, M. J. Ilamathi, and M. Nithya, "Pre-processing techniques for text mining-an overview," International Journal of Computer Science \& Communication Networks, vol. 5, no. 1, pp. 7-16, 2015.

[42] R. Fahmi Aajami, "A cognitive semantic analysis of meaning interrelationship," Arab World English Journal, vol. 10, no. 1, pp. 116-126, 2019.

[43] Y. Song, Z. Zhuang, H. Li et al., "Real-time automatic tag recommendation," in Proceedings of the 31st Annual International ACM SIGIR Conference on Research and Development in Information Retrieval - SIGIR '08, Ann Arbor, MI, USA, July 2008.

[44] A. El-Shahat, "Introductory chapter: artificial neural networks," Advanced Applications For Artificial Neural Networks, Intechopen, London, UK, 2018.

[45] S. Mirjalili, S. M. Mirjalili, and A. Lewis, "Grey wolf optimizer," Advances in Engineering Software, vol. 69, pp. 46-61, 2014.
[46] G. D. Magoulas, Y. Papanikolaou, and M. Grigoriadou, "Adaptive web-based learning: accommodating individual differences through system's adaptation," British Journal of Educational Technology, vol. 34, no. 4, pp. 511-527, 2003.

[47] E. Triantafillou, A. Pomportsis, and S. Demetriadis, "The design and the formative evaluation of an adaptive educational system based on cognitive styles," Computers \& Education, vol. 41, no. 1, pp. 87-103, 2003. 